Equipo de Cirugía Vascular y Endovascular, Servicio de Cirugía, Complejo Asistencial Barros Luco, Escuela de Postgrado, Universidad de Chile. Santiago de Chile. aResidente de Cirugía Vascular Periférica.

Apoyo financiero: No existe.

Recibido el 20 de enero de 2013, aceptado el $17 \mathrm{de}$ mayo de 2013

Correspondencia a: Dr. Gabriel Cristóbal Seguel Seguel

Sol y mar 535 edificio $E$, departamento 13, Viña del Mar, Chile.

Teléfono: + 56-9-98272823

E-mail: gabrielseguel@ gmail.com

\section{¿Por qué debemos preocuparnos del pie diabético? Importancia del pie diabético}

\author{
GABRIEL SEGUEL ${ }^{a}$
}

\section{An update on diabetic foot}

The diabetic foot is responsible for 70\% of amputations in adults, entails a significant impact in the life of patients and it is associated with an excessive use of resources in our health system. The 2009-2010 National Health Survey indicated that in the "last year" $6.7 \%$ of subjects with diabetes consulted a health care professional for diabetic foot. With the permanent rise of worldwide and national prevalence of Diabetes Mellitus and the increased life expectancy in our country, the public health system needs to adapt to this new epidemiological situation. There are interventions, which have been tested abroad, that may modify substantially the natural history of diabetic foot. However, in our country there is little experience with them. This deficiency results in increased rates of amputation and possibly a deterioration of the quality of life of diabetic patients. The objective of this article is to provide an update on the knowledge about diabetic foot.

(Rev Med Chile 2013; 141: 1464-1469)

Key words: Diabetic foot; Diabetes Mellitus; Epidemiology.
4 1 pie diabético es una enfermedad compleja responsable de $70 \%$ de las amputaciones realizadas en adultos. En Chile desconocemos su prevalencia. Este cuadro conlleva un profundo deterioro en la autovalencia, productividad y funciones psicosociales de los individuos. Para los sistemas de salud, el pie diabético y en particular la amputación, significa una importante carga económica llevándose parte significativa de los recursos totales asignados para el manejo de la diabetes mellitus (DM). Desde la perspectiva asistencial, es la principal causa de hospitalización en diabéticos. Para los equipos de Cirugía Vascular y de Cirugía General son una carga difícil de sobrellevar, tanto por los días cama, como por la cantidad de procedimientos a que deben ser sometidos. Desde otra perspectiva, intervenciones basadas en la historia natural de la enfermedad con prevención en distintos niveles ha demostrado reducir las amputaciones hasta $85 \%$. En nuestro país es reconocida la enorme carga que esta patología produce para todos los niveles de atención de salud, por lo que, desde 2006 las guías GES de diabetes mellitus y otras han intentado sin resultados controlar este problema.

\section{Diabetes mellitus}

La DM es una enfermedad crónica no transmisible de origen multifactorial. En el año 2006 la DM fue la quinta causa de muerte en el mundo con una tasa de 20,3 por 100.000 habitantes ${ }^{1}$. Se estima que 246 millones de personas en el mundo son portadoras de esta enfermedad con una prevalencia de 5,9\% en la población adulta. Al año 2025 esta cifra podría ascender a 380 millones con una prevalencia para la población adulta de $7,1 \%{ }^{2}$. En nuestro continente en el año 2000 se estimó que más de 35 millones de personas padecían diabetes mellitus, 54\% pertenecía a América Latina y el Caribe con una proyección de 64 millones al año 2025 elevándose a $62 \%$ en nuestra región ${ }^{1}$. En Estados Unidos de Norteamérica la población beneficiaria 
de Medicare presentó una prevalencia de DM de $26,4 \%$ en $2006,27,3 \%$ en 2007 y 27,9\% en $2008^{3}$.

En Chile, la encuesta nacional de salud (ENS) 2009-2010 mostró una prevalencia nacional de diabetes en el adulto de $9,4 \%$, con una prevalencia algo mayor en mujeres $(10,4 \%)$ que en hombres $(8,4 \%)$, pero no significativa ${ }^{4}$.

En Chile la expectativa de vida ascendió a 80 años en mujeres y 73 años en varones, con 11,4\% de mayores de 65 años es una de las más altas en América Latina y el Caribe, por lo tanto, nuestro sistema sanitario ha tenido que adaptarse a la nueva carga de enfermedad constituida por la DM y diseñar estrategias con efectividad demostrada a lo largo del ciclo vital ${ }^{5}$.

La DM es una de las patologías que genera mayor discapacidad y mortalidad en el adulto y adulto mayor, dato coherente con la información recabada por la primera encuesta nacional de discapacidad en que $62,9 \%$ de los encuestados declaró que su principal deficiencia es causada por una enfermedad crónica ${ }^{6}$.

Debe recordarse que frenar el aumento de la mortalidad por DM, así como mantener la tasa estandarizada por edad (14/100.000 habitantes) fueron objetivos sanitario para el decenio 20002010 lo que hasta ahora no se ha logrado ${ }^{1}$.

\section{Pie diabético}

Se denomina pie diabético a la infección, ulceración o destrucción de tejidos profundos del pie asociado con neuropatía o enfermedad arterial periférica en las extremidades inferiores de personas con diabetes ${ }^{2}$.

Ochenta y cinco por ciento de las amputaciones son precedidas por una úlcera ${ }^{7}$. Se sabe que personas con DM tienen 25 veces más riesgo que la población general de perder una de sus extremidades. Cada año más de 1 millón de personas pierde una extremidad inferior por DM. La tasa de prevalencia de amputaciones varía entre 0,2 y $4,8 \%$ con una tasa de incidencia anual que oscila entre 46,1 y 936 por 100.000 personas con $\mathrm{DM}^{2}$.

En Estados Unidos de Norteamérica la prevalencia de úlceras en el pie de pacientes diabéticos de Medicare es $8,1 \%$ en 2006, 8, $1 \%$ en 2007 y $8 \%$ en 2008. En aquellos pacientes diabéticos portadores de enfermedad arterial oclusiva la prevalencia fue de $19,2 \%$ en $2006,18,9 \%$ en 2007 y $18,6 \%$ en
2008. Así también la prevalencia de amputación de parte o toda la extremidad inferior en pacientes diabéticos beneficiarios de Medicare fue de 1,8\% en 2006, 2007 y $2008^{3}$.

Las complicaciones del pie acumulan más hospitalizaciones que cualquier otra complicación de la DM con mortalidad y morbilidad considerables $^{8}$. Las complicaciones del pie son las más serias y costosas de la DM. Estrategias que incluyen prevención, educación de pacientes y prestadores, tratamiento multidisciplinario de las úlceras y monitoreo estrecho reducen las amputaciones entre 49 y $85 \%$. Tanto la organización mundial de la salud como la International Diabetes Federation (IDF) tienen como objetivo reducir la tasa de amputaciones sobre $50 \%{ }^{9}$.

En Chile, la ENS Chile 2009-2010 ${ }^{4}$ estableció que $6,7 \%$ de los diabéticos ha tenido que consultar un profesional de la salud en el último año por pie diabético. Se estableció también que a 48,3\% de los diabéticos nunca se le había examinado los pies.

La prevalencia de las alteraciones del pie de pacientes diabéticos en Chile es desconocida, en Brasil existe el estudio de prevalencia de pie diabético desarrollado por las unidades de salud familiar en la ciudad de Recife, Pernambuco, Brasil de 2005 que utiliza como definición la dada por la $\operatorname{IDF}^{2}$ e identificó una prevalencia de $9 \%$, que osciló según el distrito sanitario estudiado en la ciudad entre 4,9 y $27,9 \%{ }^{10}$, cifras aún más altas son propuestas por Al-Maskari, quien refiere que hasta 39\% son portadores de polineuropatía, aun más, otros autores sugieren que hasta la mitad de las personas con diabetes podrían tener factores de riesgo para ulceración ${ }^{11}$.

\section{Experiencia internacional en manejo de pacientes diabéticos con problemas en el pie}

El importante consumo de recursos en esta patología y la escasa investigación existente llevó a 14 instituciones europeas especializadas en pie diabético a crear el grupo de estudio en diabetes y extremidad inferior (EURODIALE). Este grupo inició una cohorte el 1 de septiembre de 2003, siguió 1.229 pacientes por 1 año con úlcera nueva los cuales fueron reclutados en $27 \%$ en una admisión hospitalaria y en $63 \%$ en controles ambulatorios. Setenta por ciento tenía más de 10 años de DM. El grupo presentó $32 \%$ de comorbilidad disca- 
pacitante entre las que destacan impedimentos visuales $(15 \%)$, enfermedad renal crónica $(6 \%)$, insuficiencia cardiaca y angina de pecho NYHA III, IV (11\%), discapacidad de la marcha (10\%), desempleo (18\%), presencia de enfermedad arterial oclusiva en $49 \%$, polineuropatía (86\%), infección en $58 \%$ del total, en particular aquellos hospitalizados presentaron $82 \%$ infecciones. En conclusión, cualquier paciente ulcerado debe considerarse severamente enfermo, ya que esta condición se asocia a severa comorbilidad y discapacidad, lo que ayuda a comprender los resultados clínicos, utilización de recursos y calidad de vida ${ }^{12}$. En particular el grupo en que se identificó enfermedad arterial oclusiva de extremidades inferiores manifestó peores resultados tras la intervención sugerida por la IDF, lo que se profundizó en aquellos que presentaron infección ${ }^{13}$. A 8 años del inicio del estudio se ha demostrado fehacientemente que esta patología representa un enorme problema para los pacientes y el sistema de salud. El trabajo realizado por las instituciones europeas ha mostrado la posibilidad de reducir las amputaciones en $40 \%$. El riesgo de sufrir una úlcera en algún momento de la vida puede llegar a $25 \%$. En cuanto a los resultados clínicos 77\% de los pacientes curó (con o sin amputación menor), $5 \%$ requirió amputación mayor y $6 \%$ murió. Dieciocho por ciento requirió de una amputación menor para cicatrizar, de las cuales $55 \%$ fueron de ortejo, $34 \%$ un ortejo y parte del metatasiano correspondiente y $11 \%$ del medio pie. La alta tasa de amputaciones menores se correlacionaba con el score de severidad de enfermedad. En el resultado principal, curación de la úlcera, no hubo diferencia al comparar pies sin enfermedad arterial oclusiva infectados y no infectados, sin embargo, pese a este caso particular hubo una tendencia clara a empeorar los resultados en curación de úlcera, amputación mayor, menor y muerte según se producía el cambio entre sin enfermedad arterial oclusiva sin infección, sin enfermedad arterial oclusiva con infección, con enfermedad arterial oclusiva sin infección, con enfermedad arterial oclusiva con infección ${ }^{14}$. Estos resultados son concordantes con el grupo de Real Collado quien estudió los factores de riesgo asociados a amputación en pacientes diabéticos, encontrando como factores más relevantes de amputación y además predictores independientes: Amputación previa $(\mathrm{OR}=3,7)$, enfermedad arterial oclusiva $(\mathrm{OR}=7,1)$ e infección grave $(\mathrm{OR}=14,4)^{15}$.
En Latinoamérica existe "The Brazilian save the diabetic foot Project"16 que desde su inicio en 1992 con la participación del diabetólogo, enfermera, asistente de enfermería y trabajadora social, basando su actuación en la experiencia británica previamente publicada, logró disminuir progresivamente las amputaciones hasta en $77,8 \%$ en mujeres. Estos resultados han llevado al crecimiento del recurso humano y complejidad de las prestaciones hasta llegar en el año 2002 a un equipo multidisciplinario en que se agregan varias especialidades médicas, fisioterapeutas, ortesistas y otros.

\section{Realidad chilena}

No existen indicadores respecto del manejo del pie diabético en nuestro país, sin embargo, en el año 2010 se publicó el informe final de la evaluación sanitaria de las intervenciones GES para diabetes mellitus ${ }^{17}$, en este documento se indica que la meta incluida en Healthy People 2010 es reducir la tasa de amputaciones en extremidades inferiores a 1,8/1.000 diabéticos. En Chile se realizan más de 2.000 amputaciones cada año por DM. La tasa de amputación en población diabética es un indicador usado internacionalmente; debe considerarse de tipo proxy, ya que, nos aproxima a un fenómeno, pero no lo mide directamente. $\mathrm{Da}$ cuenta de la calidad del manejo ambulatorio de la DM. A nivel nacional la tasa de amputaciones en población diabética aumentó de 3,5 en 2002 a 4,5/1.000 diabéticos en 2006 lo que corresponde a un incremento de $28 \%$.

\section{Evaluaciones económicas de intervenciones en pie diabético}

El costo de tratar una úlcera en el grupo EURODIALE fue de $€ 8.000$ la mayor parte de esta cifra relacionada con las hospitalizaciones por lo cual se torna atractivo invertir en su tratamiento ambulatorio. Los mayores costos se alcanzaron en pacientes que requirieron amputación de una extremidad ascendiendo hasta $€ 5.222$. El costo de tratamiento fue significativamente mayor en los pacientes portadores de enfermedad arterial oclusiva. Estrategias que apunten a referencia precoz para el tratamiento antibiótico adecuado 
e intervención por cirugía vascular ayudan a prevenir la pérdida de tejido y reducen las rehospitalizaciones y $\operatorname{costos}^{14}$.

El costo estimado de las úlceras y amputaciones para los pagadores del sistema de salud de Estados Unidos de Norteamérica fue de USD 10,9 billones en 2001, usando la misma metodología para el caso del Reino Unido fue de 252 millones de libras. En una revisión de estudios entre 1994 y 2000, tras ajuste por inflación el costo de cerrar úlceras que no requirieron amputación varió entre USD 993 y USD 17.519 (equivalencia año 1998). En una revisión de evaluaciones de costos para lesiones del pie el costo estimado para un paciente amputado varía entre USD 16.488 y USD 66.215 (equivalentes año 1998). En consecuencia la prevención de úlceras y amputaciones en pacientes diabéticos debe ser el objetivo más importante para una utilización más eficiente de los recursos ${ }^{18}$.

La relevancia de la discapacidad física producida por DM llevó al Ministerio de Salud de Chile a proponer su reducción como uno de los objetivos sanitarios de la década recién pasada ${ }^{1}$.

La investigación en cuidados del pie de pacientes diabéticos y la implementación de programas de prevención es una de las formas más costo efectivas de provisión de cuidados de salud ${ }^{1}$.

Mc Cabe y cols reportaron una evaluación económica de ensayo clínico en que comparan una atención habitual en una clínica ambulatoria de diabetes con un programa de screening y protección del pie que incluyó Semmes Weinstein, biotensiómetro y palpación de pulsos, tras encontrar alteraciones en estas pruebas se repitió la evaluación y se agrega índice tobillo brazo, medición transcutánea de oxígeno y radiografías del pie para evaluar puntos de apoyo. Tras lo anterior se realizaron cuidados del pie, medidas de higiene, calcetines, calzado de protección y seguimiento por 2 años. La intervención demostró ser costo efectiva en reducir amputaciones mayores, sin embargo, no fue capaz de demostrar costo efectividad en cierre de úlceras y amputaciones menores ${ }^{19,20}$.

En un análisis de costo utilidad, Ragnarson comparó la estrategia en curso (año 1998) con una prevención óptima tomada de las recomendaciones IDF que incluía educación de pacientes y prestadores, provisión de cuidados del pie, calzado apropiado y manejo por equipos multidisciplinarios. Se realizó simulación en modelo de Markov con horizonte a 5 años. Considerando la intervención costo efectiva si puede reducir la incidencia úlceras y amputaciones en $25 \%$, siendo válido esto para todos los pacientes diabéticos con factores de riesgo. Realizaron 3 cohortes de edad, y análisis de sensibilidad de una vía para diversas variables, entre ellas tasa de descuento, incidencia de úlceras, amputación de extremidad inferior, resultando la prevención óptima dominante en casi todas las alternativas ${ }^{21}$.

Un programa efectivo de cuidados del pie debe tener un equipo multidisciplinario. En los últimos 15 años se ha demostrado que el cuidado del pie de pacientes diabéticos es óptimo sólo cuando el financiamiento y recursos humanos son suficientes. El abordaje multidisciplinario ha mostrado una reducción de amputaciones desde $49 \%$ hasta $85 \%^{2}$.

\section{Calidad de vida y pie diabético}

En 2010 un grupo noruego publicó diferencias significativas en calidad de vida medida con EQ5D entre diabéticos tipo 2 sin complicaciones (media índice $\mathrm{EQ}=0,85$ ), con una complicación (media índice $\mathrm{EQ}=0,8$ ) y con dos o más (media índice $\mathrm{EQ}=0,64)$; además presentó una media para el índice $\mathrm{EQ}$ de 0,81 ( $\mathrm{DS}=0,22$ ) en los pacientes portadores de DM tipo 2 incorporados al estudio ${ }^{22}$. García Morales y cols ${ }^{23}$ estudiaron la calidad de vida relacionada con salud usando SF-36 en diabéticos con y sin pie diabético, encontrando una disminución significativa en la calidad de vida en pacientes portadores de esta complicación. Siendo los factores que más impactan en la calidad de vida la neuropatía periférica, historia de amputación y mal control metabólico. Llama la atención un impacto mayor en la calidad de vida de las mujeres que en los hombres portadores de pie diabético, así como, la ausencia de impacto de la enfermedad arterial oclusiva de extremidades inferiores (en la calidad de vida).

Existe evidencia respecto de que un enfoque preventivo y un tratamiento precoz de las úlceras del pie son costo efectivas, mejora la calidad de vida de los pacientes, ahorra recursos y aumenta la sobrevida ${ }^{24,25,26}$.

En conclusión, es perentorio que autoridades políticas, directivos, clínicos y pacientes asuman el desafío de optimizar el manejo del pie diabético, ya que, el alza progresiva en la prevalencia de DM en 
el mundo y particularmente en Chile sugiere que la brecha de atención y calidad para esta patología se profundizará. Para esto, el desarrollo de unidades especializadas en pie diabético que apliquen las mejores prácticas clínicas, multidisciplinarias, con recursos suficientes y capacidad de gestión adecuada parece ser el camino a seguir para optimizar nuestros resultados, la costo efectividad de las intervenciones y la calidad de vida de nuestros pacientes.

\section{Referencias}

1. Minsal. Guía clínica Diabetes Mellitus tipo 2, 2006. Disponible en www.redsalud.gov.cl [Consultado el 11 de enero de 2013].

2. International Working Group on the Diabetic Foot. International consensus on the diabetic foot and practical guidelines on the management and prevention of the diabetic foot [CD-ROM]; 2007.

3. David J, Scot M, Ole J, Charles E, Thomas M, López $\mathrm{K}$, et al. Prevalence of diabetes, diabetic foot ulcer, and lower extremity amputation among Medicare beneficiaries, 2006 to 2008. Data points publications series. 2011. Disponible en: http://effectivehealthcare.ahrq.gov [Consultado el 11 de enero de 2013].

4. Minsal. Encuesta Nacional de Salud ENS Chile 20092010; 2011. Disponible en http://www.minsal.gob.cl [Consultado el 11 de enero de 2013].

5. DEIS. Mortalidad de ambos sexos según las principales causas específicas de defunción 2009. Disponible en: http://webdeis.minsal.cl/estadisticas-mortalidad [consultado el 11 de enero de 2013].

6. INE. Primer estudio nacional de la discapacidad en Chile; 2004. http://www.ine.cl [Consultado el 11 de enero de 2013].

7. Apelqvist J. Diagnostics and treatment of the diabetic foot. Endocrine 2012; 42: 384-97.

8. Cheer K, Shearman C, Jude E. Managing complications of the diabetic foot. BMJ 2009; 339: 1304-7.

9. Bakker K, Apelqvist J, Schaper N. Practical guidelines on the management and prevention of the diabetic foot 2011. Diabetes Metab Res Rev 2012; 28 (suppl 1): 22531.

10. Ramos I, Viera W, Freese E, Wanderley M, De Lira M, Santos P. Prevalence of diabetic foot and associated factors in the family health units of the city of Recife, Pernambuco State, Brazil, in 2005. Cad Saude Publica 2008; 24: 2861-70.

11. Al-Maskari F, El-Sadig M. Prevalence of risk factors for diabetic foot complications. BMC Family practice 2007; 8: 59-67.

12. Prompers L, Huijberts M, Apelqvist J, Jude E, Piaggesi A, Bakker K, et al. High prevalence of ischaemia and serious comorbidity in patients with diabetic foot disease in Europe. Baseline results from Eurodiale study. Diabetologia 2007; 50: 18-25.

13. Prompers L, Schaper N, Apelqvist J, Edmonds M, Jude E, Mauricio D, et al. Prediction of outcome in individuals with diabetic foot ulcer: focus on the differences between individuals with and without peripheral arterial disease. The EURODIALE Study. Diabetologia 2008; 51: 747-55.

14. Schaper N. Lessons from eurodiale. Diabetes Metab Res 2012; 28 (suppl 1): 21-6.

15. Real J, Valls M, Basanta M, Ampudia F, Ascaso J, Carmena R. Estudio de factores asociados con amputación en pacientes diabéticos con ulceración en pie. An Med Interna 2001; 18: 59-62.

16. Pedrosa H, Leme L, Novaes C, Saigg M, Sena F, Gomes E, et al. The diabetic foot in South America: Progress with the Brazilian Save the Diabetic Foot Project. International Diabetes Monitor 2004; 4: 10-7.

17. Universidad Mayor, Escuela de Salud Pública. Evaluación sanitaria de las intervenciones GES. Diabetes Mellitus tipo $2 ; 2010$.

18. Boulton A, Vileikyte L, Ragnarson G, Apelqvist J. The global burden of diabetic foot disease. Lancet 2005; 366 : 1719-24.

19. NICE. Clinical guidelines for type 2 Diabetes. Prevention and management of foot problems. 2003. http://www. nice.org.uk [Consultado el 11 de enero de 2013].

20. Mc Cabe C, Stevenson R, Dolan A. Evaluation of a Diabetic Foot Screening and protection program. Diabet Med 1998; 15: 80-4.

21. Ragnarson G, Apelqvist J. Prevention of diabetes-related foot ulcers and amputations: a cost-utility analysis base don markov model simulations. Diabetología 2001; 44: 2077-87.

22. Solli O, Stavem K, Kristiansen I. Health related quality of life in diabetes: The associations of complications with EQ5D scores. Health Qual Life Outcome 2010; 8: 1-8.

23. García-Morales E, Lázaro-Martínez J, MartínezHernández D, Aragón-Sánchez J, Beneit-Montesinos J, González-Jurado M. Impact of Diabetic Foot Related Complications on the Health Related Quality of Life (HRQoL) of Patients- A Regional Study in Spain. Int J low Extrem Wounds 2011; 10: 6-11.

24. Habib SH, Biswas KB, Akter S, Saha S, Ali L. Costeffectiveness analysis of medical intervention in patients 
Pie diabético - G. Seguel

with early detection of diabetic foot in a tertiary care hospital in Bangladesh. J Diabetes Complications 2010; 24: 259-64.

25. Ortegon M, Redekop W, Niessen L. Cost-effectiveness of prevention and treatment of the diabetic foot: a Markov analysis. Diabetes Care 2004; 27: 901-7.

26. Girod I, Valensi P, Laforêt C, Moreau-Defarges T, Guillon P, Baron F. An economic evaluation of the cost of diabetic foot ulcers: results of a retrospective study on 239 patients. Diabetes Metab 2003; 29: 269-77. 